\title{
Genetic and Environmental Interplay in Adolescent Substance Use Disorders
}

\author{
Lindsey A. Hines ${ }^{1} \cdot$ Katherine I. Morley $^{1} \cdot$ Clare Mackie $^{1} \cdot$ Michael Lynskey ${ }^{1}$
}

Published online: 3 April 2015

(C) Springer International Publishing AG 2015

\begin{abstract}
Adolescent substance use is of considerable public health importance. This narrative review provides a brief background to genetically informative research methodologies and highlights key recent literature examining the interplay between genetic and environmental influences in the etiology of substance use. Twin studies have quantified the magnitude of genetic and environmental influences, and more recently, corelative and Children of Twin designs have shown environments can moderate heritability. Studies have identified a number of specific gene variants (e.g. OPRM1, DRD4, 5HTTLPR) that interact with parenting and peer influence, and the effectiveness of interventions may vary by genotype. However, little research has taken into account the stage-sequential nature of substance use. This may obscure important differences in the genetic and environmental influences, and their interplay, at the stages of escalation to problem use. Future research needs to build on existing methodologies to disentangle the complexities of progression in adolescent substance use.
\end{abstract}

This article is part of the Topical Collection on Adolescent Substance Abuse

Michael Lynskey

michael.lynskey@kcl.ac.uk

Lindsey A. Hines

Lindsey.hines@kcl.ac.uk

Katherine I. Morley

Katherine.morley@kcl.ac.uk

Clare Mackie

Clare.mackie@kcl.ac.uk

1 Addictions Department, King's College London Institute of Psychiatry, Psychology and Neuroscience, 4 Windsor Walk, London SE5 8BB, UK
Keywords Adolescence $\cdot$ Gene $\times$ environment interaction . Substance use $\cdot$ Genetic $\cdot$ Environmental

\section{Introduction}

Substance use is an area of considerable public and research importance due to the associated health and social consequences [1]. Use during adolescence is an area of particular research focus, not just because adolescence is the typical period for substance use initiation [2] but also because of the acute risks and harms [3-5] from substance use and the strong association between onset, extent of use during adolescence and risks for the subsequent development of substance dependence and related harms [6-12]. There has been a long history of research into environmental risk factors for the initiation of substance use [13] and more recently a recognition of strong genetic influences on substance use and substance use disorders, derived principally from the findings of twin studies [12, 14-18]. Advances in genotyping technologies have permitted the expansion of research on these genetic influences to the identification of specific genetic variants associated with drug use. Despite this, relatively few genetic variants have been identified as being robustly associated with substance dependence, with some notable exceptions [19-21].

The limited number of genetic variants with strong, robust associations with substance use has led to increasing focus on the interplay between genetic predispositions and environmental exposures in the etiology of substance use and escalation to problems [22-26]. Gene by environmental interplay encompasses both gene by environment interaction and gene by environment correlation. Three main categories of gene by environmental correlation have been identified. These are active correlation, whereby individuals select, modify or construct experiences that are correlated with their genetic 
predisposition; passive correlation, whereby individuals are passively exposed to environments that are correlated with their genetic predispositions; and evocative correlation, whereby the individual's genotype elicits a certain response from the environment around them [27]. Gene by Environment Interactions $(G \times E)$ occur when environmental effects on individuals vary by genotype or when the environment alters the effect a gene has on an individual's physiology [27, 28].

In this review, we focus on the recent literature exploring genetic and environmental influences on the development of substance use and dependence during adolescence. By highlighting methodological approaches, preliminary findings and the necessity of considering the stage-sequential nature of substance use, we identify areas that show the greatest promise for disentangling this complex etiology.

\section{Genetically Informative Research and the Risk Factors for Adolescent Substance Use}

The magnitude of genetic and environmental influences on substance use disorders has been broadly quantified by twin studies. Agrawal and Lynskey [17] noted that estimates of the heritability of substance dependence ranged from 0.30 to 0.70 and were broadly equivalent across individual substances. Twin methodologies allow the quantification of the magnitude of both shared environmental influences that increase twin similarity on a trait and non-shared environmental influences that do not increase similarity on a trait [27]. Shared genetic influences are typically less prominent than non-shared environmental influences in the etiology of substance dependence; although in adolescence, the shared environment may be relatively more prominent. In addition to quantifying the magnitude of genetic and shared environmental influences on substance dependence, twin studies have also demonstrated that there are likely to be genetic influences that form a vulnerability to substance use across drug classes [29, 30], although some evidence has been found for separate, albeit highly correlated, licit and illicit drug genetic factors [31].

\section{Utilising Traditional Twin Studies to Explore Gene by Environment Interplay}

Twin studies have not only allowed quantification of the magnitude of genetic effects but have also provided strong evidence that the magnitude of genetic influences is altered by the environment. For example, Boardman et al. have demonstrated the impact of public health policies, finding that the magnitude of genetic influences on smoking desistance increased following the introduction of restrictive legislation on smoking behaviours [32] and that genetic influences on daily smoking in adolescents were lower in states with more restrictive access to tobacco products [33]. Social factors also play an important role. For example, the heritability of smoking is higher in schools where the most popular students smoke [34]; genetic influences on adolescent smoking are more prominent in the presence of low parental monitoring [35]; and heritable influences on adolescent substance use are more prominent in environments characterised by high levels of peer substance use [36].

\section{The Utility of Large, Linked Administrative Data Sets}

In addition to results from twin studies, Kendler and his colleagues have recently used a novel research strategy to examine genetic influences, based on the analysis of whole population administrative data sets from Sweden, with sample sizes exceeding one million individuals. Combining official records of treatment seeking, official police contact and related information at a whole population level with information on outcomes in relatives (e.g. full siblings, cousins), they have confirmed substantial heritability for drug abuse in both males $(55 \%)$ and females $(73 \%)$, with environmental factors shared by siblings operating only in males [37]. The convergence of these findings with those from traditional twin studies, which have typically studied less severe phenotypes based on selfreport symptom data, is impressive. Using a similar approach to examine concordance for drug abuse in pairs of related individuals, the authors demonstrated the importance of family environmental influences on drug abuse in sibling pairs, where those whose older sibling had recorded drug abuse had a 1.42 (95\% CI, 1.31-1.54) increased likelihood of drug abuse compared to those whose younger siblings had recorded drug abuse [38••]. Similar findings were reported for cousin pairs, who were found to be significantly more similar in recorded drug abuse if they were close in age and location when growing up [39].

In addition to confirming the importance of both (latent) genetic and environmental influences on risks for drug abuse, these authors have presented a number of analyses examining the extent to which specific, measured environmental exposures are associated with risks for drug abuse while controlling for heritable influences on such risk. For example, Giordano et al. [40], using a co-relative comparison within sibling and cousin pairs discordant for exposure to trauma, demonstrated that experiencing a second-hand traumatic event (e.g. having a parent or sibling be assaulted or die) before age 15 was associated with twice the risk for the development of drug abuse. They used a similar approach to highlight the importance of neighbourhood social deprivation on the development of drug abuse [41]. Kendler et al. [42] highlighted risks for drug abuse associated with exposure to both peer deviance and parental divorce while also implicating interactions between genetic liability for drug abuse and peer deviance and between parental divorce and peer deviance in the etiology of officially recorded drug abuse. For those with low genetic risk (determined by the 
drug abuse records of relatives), living in an area with high peer deviance was associated with an increase in 28.1 cases of drug abuse per 10,000 person-years. In comparison, being at very high genetic risk in a high risk area was associated with an increase of 78.0 cases per 10,000 person-years.

\section{The Use of Children of Twins Design}

One potential liability of the traditional twin method is that any $\mathrm{G} \times \mathrm{E}$ will be confounded with, and therefore inflate, estimates of heritability unless measured environmental exposures are explicitly modelled [43]. One design which can model both main effects of genes and environment and gene by environmental interactions is the children of twins (CoT) design. This design allows a comparison of outcomes in (1) children at high genetic risk and high environmental risk (e.g. parent is alcohol dependent), (2) high genetic risk but reduced environmental risk (parent is not alcohol dependent but their MZ co-twin is), (3) intermediate genetic risk but reduced environmental risk (parent is not alcohol dependent but their DZ co-twin is) and (4) children at low genetic and low environmental risk (control families; both parent and co-twin are not alcohol dependent). Thus, it allows detection not only of genetic transmission but also environmental consequences of parental alcohol dependence that may depend upon offspring genotype (Gene $\times$ Shared Environment interaction [43]) or be masked by genetic nonadditivity.

Applying the CoT design to study the links between parental drug dependence (alcohol or cannabis) and onset of substance use in offspring, Waldron et al. [44••] reported that both children of parents who were drug dependent (high genetic/ high environmental risk) and those of non-drug-dependent parents whose MZ co-twin was drug dependent (high genetic/low environmental risk) had elevated rates of early onset tobacco ( 7.30 and 2.22 times higher risk, respectively), alcohol (1.43 and 2.82 times higher risk, respectively) and cannabis use (only significant for those with high genetic/ low environmental risk, where it was 3.03 times higher). While this pattern of results strongly implicates heritable influences in transmission of risks associated with parental drug dependence, further analyses also highlighted that, independent of these influences, exposure to parental divorce significantly elevated risks of early onset substance use.

\section{Interactions of Specific Genetic Variants with Environmental Exposures}

While the research above has focussed on latent genetic influences, research that has explored specific genes in relation to the environment has also found encouraging results for $\mathrm{G} \times \mathrm{E}$. There are a number of considerations when selecting specific gene variants to be tested against specific environmental exposures. What is considered a large sample for environmental research may be underpowered for genetic studies, with extremely high numbers of participants required to identify strong $\mathrm{G} \times \mathrm{E}$ between genotypes and environmental exposures that commonly occur $[45,46]$, resulting in the employment of prioritisation strategies for investigation of $\mathrm{G} \times \mathrm{E}$ that focus on genetic variants that are common in the population; that are associated with the disorder being studied; or that are associated with individual response to the environmental factor under consideration $[28,45]$. Consequently, the genetic variants that have been studied to date are those commonly studied in the mental health $\mathrm{G} \times \mathrm{E}$ literature.

There is some evidence that the influence of specific gene variants may be moderated by environmental exposures such as neighbourhood peer substance use, parental supervision, and parent-child attachment. For example, Daw et al. [47] studied the influence of 5HTTLPR in combination with measures of peer smoking. There was not a statistically significant main effect of neighbourhood peer cigarette use, but those with more copies of the 5HTTLPR short allele had a significantly higher hazard of smoking initiation in environments where a higher proportion of neighbourhood peers smoked. Specifically, the hazard ratio for the interaction between peer use and number of 5HTTLPR short alleles on any smoking was $3.532(P=0.002)$ and $5.686(P<0.001)$ for regular smoking. In addition, COMT has been found to interact with parental supervision to affect frequency of drinking at age 19 and with parental involvement to affect amount consumed at age 19 [48]. The interaction between OPRM1 and parental rule setting was also found to significantly differentiate heavy drinkers from light drinkers [49]. Further, the effect of an ADH1B variant has been found to be moderated by the drinking behaviour of peers, with a protective effect on reaching drinking milestones observed when no friends were reported to be drinking that was significantly reduced when most or all best friends were reported to be drinking [50]. Finally, higher numbers of DRD4 alleles significantly interacted with parentchild attachment to increase risk of problematic smoking (anxious attachment) and cannabis use (avoidant and anxious attachments) [51].

\section{Genetic Variants and Response to Substance Use Interventions}

A new and emerging research area is the examination of how specific genetic variants may alter response to treatment or prevention interventions. Understanding why certain interventions are successful for some people and less so for others would allow for the development of tailored intervention programmes and represent an example of potential $\mathrm{G} \times \mathrm{E}$ interactions. However, only recently has research begun to consider whether an individual's genetic risk can moderate the effects of participation in an intervention for substance use. 
Brody et al. [52••] reported the results of two longitudinal family-centred randomised controlled trials designed to reduce alcohol use in 900 11-17 year olds. They examined whether adolescents assigned to the intervention condition who carried a dopaminergic or GABAergic susceptibility gene would demonstrate greater decreases in alcohol use than adolescents who did not carry either susceptibility gene. They found that participants within the intervention condition carrying two or three susceptibility genes evinced smaller increases in alcohol use than participants with none or only one susceptibility gene. Regression estimates for the interaction between gene and intervention (significant after adjustment for multiple comparisons) were -0.86 (SE 0.22) for GABRG1, -0.67 (SE 0.21) for GABRA2 and -1.19 (SE $0.29)$ for DRD2. These findings provide support for the differential susceptibility theory [53] which suggests that the genetic make-up of some individuals may increase malleability or susceptibility to both negative and positive environmental influences. Thus, some individuals may be adversely affected by negative exposures and may also be more likely to benefit from positive environments. Therefore, interindividual variability can exist in response to positive experiences such as programmes designed to address substance use.

\section{Methodological Challenges of Specific Genetic Variants}

Research is providing promising results for $\mathrm{G} \times \mathrm{E}$ and has highlighted genes that warrant further exploration. However, the selection of specific genetic variants for study has limitations. With regard to the environmental exposures selected for study, there are an extensive number of potential exposures that can be combined with the plethora of gene candidates. Sher and colleagues [54] have highlighted that the unavailability of information on the number of possible environmental influences means that there is no "environome" to compare with the genome. Distal factors that are thought to have a long-term effect (such as childhood family environment) can be differentiated from proximal exposures (such as availability), and studies of $\mathrm{G} \times \mathrm{E}$ in adolescent substance use may benefit from systematically classifying environment in this way. There is also a need to identify whether $\mathrm{G} \times \mathrm{E}$ findings remain stable at different time points. Developmental stage has previously been shown to affect gene associations [55], and specifically for adolescent substance use, it is important to confirm whether associations differ depending on the type of drug, the stage of substance use, the developmental stage of the tested population and across subgroups. Finally, despite years of candidate gene studies, there are still few replicated associations [46]. Additional lines of $\mathrm{G} \times \mathrm{E}$ research may identify more robust associations.

\section{Polygenic Risk Scores}

Focussing research on combinations of genes or gene systems may be a more efficient strategy for understanding $\mathrm{G} \times \mathrm{E}$ in substance use. The advent of genome-wide association studies (GWAS) has implicated multiple genes from multiple biological pathways in the etiology of substance use $[19,56]$. However, as for other complex traits, the effect sizes of individual gene variants associated with substance use are small and fall short of accounting for the expected amount of variability based on heritability estimates [57]. Taking this into account, some researchers have moved from examining single genes for $\mathrm{G} \times \mathrm{E}$ to considering polygenic risk scores $[58 \bullet, 59,60]$. In principle, these scores work in the same way as other risk prediction models, aggregating the effects of multiple genetic variants to produce a quantitative estimate of (genetic) risk for a particular trait for each individual.

While risk scores do have the potential to be useful and informative, careful consideration must be given to (i) methods used to develop these scores, (ii) the strength of association between the polygenic score and the outcome(s) of interest and (iii) whether the results are applicable beyond the study sample [61]. An illustrative paper on this topic used a longitudinal community sample to investigate the interaction between a polygenic risk score and age in relation to tobacco consumption [62•]. The authors demonstrated that, even when multiple genetic factors were combined, the polygenic score only accounted for a small proportion of the variance in a trait for nicotine (in their example, from 0.1 to $1 \%$ of the variance in cigarette consumption between the ages of 14 and 24) and did not show the same relationship for alcohol. This does not rule out the use of polygenic risk scores but does serve to underline the need for realistic expectations about their likely predictive power and the importance of considering their potential contribution in combination with other, nongenetic, risk factors [63].

\section{Considering the Stage-Sequential Nature of Drug Dependence}

There are three key features of the relationship between genetic and environmental influences on the development of substance use and dependence that require further elaboration. Firstly, it is important to note that the development of substance dependence is a stage-sequential process in which a number of different transitions must first occur (see Fig. 1). Many individuals will reach different stages without progressing to dependence, but traditionally, most studies of dependence have conflated these stages. For example, the common comparison of individuals who are substance dependent against those who are not dependent often fails to distinguish between non-cases who may have never used a drug and 
Fig. 1 Diagram of stagesequential drug use, depicting plausible stage-specific genetic and environmental influences, and the increasing magnitude of genetic influences on later stages

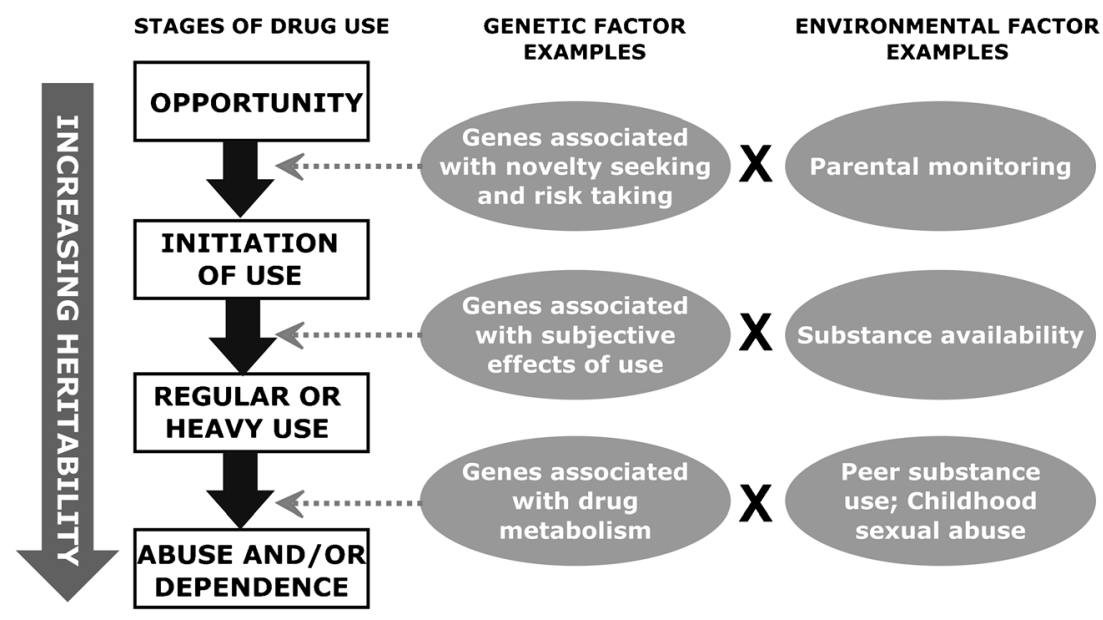

those who used the drug (potentially regularly) but did not progress to dependence. There is also value in exploring variation amongst those who reach different stages, differentiating not only those who do or do not initiate use but also those who initiate use earlier or later [64]. Despite research highlighting the necessity of considering early substance use stages, this has rarely been considered, making it unclear at which stages in the development of drug dependence specific genetic or environmental influences are most prominent [65].

Secondly, research suggests that the relative strength of genetic and environmental influences on the development of substance dependence varies by both stage of substance use and by developmental age [12, 66-70]. For example, there is evidence that, in their aggregate, genetic influences are relatively weaker in younger aged samples [71] and at earlier stages (e.g. initiation) in the development of substance dependence [16] (see Fig. 1). Nonetheless, there is also evidence of substantial, albeit incomplete, overlap in the genetic influences on initiation and problem use [72-74].

A third feature that deserves further exploration is the extent to which the importance of measured genetic and environmental influences on substance dependence may vary across stages of substance involvement (see Fig. 1). Early stages of substance use, such as initiation, might be genetically influenced through personality traits such as novelty seeking [75], while genetic factors associated with sensitivity to drug effects may influence progression of use. At subsequent stages, such as drug dependence and withdrawal, genetic influences on drug metabolism may have stronger associations. Similarly, there may be distal and proximal environmental risk factors that are unique to specific stages of substance involvement, while others may act across multiple stages or show correlation while not being identical [72]. Research is also emerging into how external influences can lead to changes in gene function at a cellular level [76], and these epigenetic mechanisms have recently begun to attract interest in the field of substance use [77-79]. Speculatively, early stages of use may be influenced by pre-existing epigenetic modification (resulting, for example, from childhood stressors [80-83]) with later stages influenced by epigenetic modifications brought about by substance use. Consequently, it is expected that the use of the stage-sequential approach will reveal that observed genetic and environmental associations will alter throughout the sequence towards dependence.

Recent research utilising the stage-sequential approach has demonstrated differences in association by stage of use for both environmental and genetic factors. For example, Sartor et al. [68] reported a number of associations with environmental factors that were unique to onset of alcohol use (e.g. male gender, attention deficit hyperactivity disorder, parental divorce and maternal alcohol dependence), while others were unique to the transition from alcohol use to dependence (e.g. nicotine dependence, cannabis abuse, generalised anxiety disorder). Similar results have been obtained for genetic risk factors. Belsky et al. [58•] reported that a multi-locus genetic risk score, derived from the results of metaanalyses for nicotine dependence, was unrelated to initiation of tobacco use but was significantly associated with increased risks for daily tobacco use, more rapid progression from initiation to heavy use, increased risks for the development of nicotine dependence and reduced likelihood of successful cessation. The literature on substance use behaviour trajectories has typically studied behaviour change that occurs between substance use stages, and modelling development and changes in substance use over time has found that GABRA2 is associated with an increase in drunkenness between ages 18-19, suggested to be due to the enhanced independence related to reaching adulthood [84]. Similarly, OPRM1 has been found to differentiate those who were light drinkers from those who had progressed to moderate drinking in participants followed up over 6 years (participants on average aged 14.3 at start of study) [49]. 
In summary, studies utilising the stage-sequential approach are likely to reveal differential genetic influences at different stages, with substance-specific genes more likely to operate at later stages [85]. Different environmental influences are also likely to operate at each stage, and research into trajectories suggests that environmental change should be incorporated into analysis [84]. Taking this approach to research is key for gaining a comprehensive understanding of $G \times E$ in the development of adolescent substance use and to reaching meaningful conclusions about opportunities for intervention.

\section{Conclusions}

The research currently available highlights the role of genetic and environmental influences and, importantly, their interplay in the development of adolescent substance use and provides intriguing avenues for potential interventions. The evidence shows promising leads with regard to gene variants that warrant further exploration, the composite effects of genes on substance use and the importance of the type and timing of environmental influences in shaping adolescent substance use behaviour.

Genetic and environmental interplay continues to be a promising avenue for exploration in order to understand the underlying causes of adolescent substance use and progression and future research will need to apply the optimal strategies for investigating this area [28, 45, 61], demonstrate the replicability of findings and overcome issues around the measurement of phenotypes. Incorporation of new methodologies provides the best opportunity to overcome the barriers to these aims. As discussed in this review, the innovative use of noninvasive methods for research through administrative data sets [38••, 39, 41, 42] shows promise for analysis of large samples with measured phenotypes at relatively low cost and effort. Combining such methods with a stage-sequential approach to understanding genetic and environmental influences on drug involvement and response to intervention may be key to disentangling the complex etiology of one of the great public health issues of our time.

\section{Compliance with Ethics Guidelines}

Conflict of Interest Lindsey A. Hines, Katherine I. Morley, Clare Mackie and Michael Lynskey declare that they have no conflict of interest.

Preparation of this manuscript was partially supported by DA023668 and AA021492 from the US NIH.

Human and Animal Rights and Informed Consent This article does not contain any studies with human or animal subjects performed by any of the authors.

\section{References}

Papers of particular interest, published recently, have been highlighted as:

- Of importance

• Of major importance

1. Degenhardt L, Whiteford H, Hall WD. The global burden of disease projects: what have we learned about illicit drug use and dependence and their contribution to the global burden of disease? Drug Alcohol Rev. 2014;33(1):4-12.

2. Kandel DB, Logan JA. Patterns of drug use from adolescence to young adulthood: I. Periods of risk for initiation, continued use, and discontinuation. Am J Public Health. 1984;74(7):660-6.

3. Movig KL et al. Psychoactive substance use and the risk of motor vehicle accidents. Accid Anal Prev. 2004;36(4):631-6.

4. Tapert SF et al. Adolescent substance use and sexual risk-taking behavior. J Adolesc Health. 2001;28(3):181-9.

5. Hingson RW, Zha W, Weitzman ER. Magnitude of and trends in alcohol-related mortality and morbidity among U.S. college students ages 18-24, 1998-2005. J Stud Alcohol Drugs Suppl. 2009;2009(16):12-20.

6. Hingson RW et al. Age of drinking onset and injuries, motor vehicle crashes, and physical fights after drinking and when not drinking. Alcohol Clin Exp Res. 2009;33(5):783-90.

7. Lynskey MT et al. Early-onset alcohol-use behaviors and subsequent alcohol-related driving risks in young women: a twin study. J Stud Alcohol Drugs. 2007;68(6):798-804.

8. Grant JD et al. Associations of alcohol, nicotine, cannabis and drug use/dependence with educational attainment: evidence from cotwincontrol analyses. Alcohol Clin Exp Res. 2012;36(8):1412-20.

9. Hingson RW, Heeren T, Winter MR. Age at drinking onset and alcohol dependence: age at onset, duration, and severity. Arch Pediatr Adolesc Med. 2006;160(7):739-46.

10. Chen CY, Storr CL, Anthony JC. Early-onset drug use and risk for drug dependence problems. Addict Behav. 2009;34(3):319-22.

11. Behrendt $\mathrm{S}$ et al. Transitions from first substance use to substance use disorders in adolescence: is early onset associated with a rapid escalation? Drug Alcohol Depend. 2009;99(1-3):68-78.

12. Lynskey MT et al. An Australian twin study of cannabis and other illicit drug use and misuse, and other psychopathology. Twin Res Hum Genet. 2012;15(5):631-41.

13. Hawkins JD, Catalano RF, Miller JY. Risk and protective factors for alcohol and other drug problems in adolescence and early adulthood: implications for substance abuse prevention. Psychol Bull. 1992;112(1):64-105.

14. Lynskey MT et al. Genetic and environmental contributions to cannabis dependence in a national young adult twin sample. Psychol Med. 2002;32(2):195-207.

15. Verweij KJ et al. Genetic and environmental influences on cannabis use initiation and problematic use: a meta-analysis of twin studies. Addiction. 2010;105(3):417-30.

16. Lynskey MT, Agrawal A, Heath AC. Genetically informative research on adolescent substance use: methods, findings, and challenges. J Am Acad Child Adolesc Psychiatry. 2010;49(12):1202-14.

17. Agrawal A, Lynskey MT. Are there genetic influences on addiction: evidence from family, adoption and twin studies. Addiction. 2008;103(7):1069-81.

18. Kendler KS. What psychiatric genetics has taught us about the nature of psychiatric illness and what is left to learn. Mol Psychiatry. 2013;18(10):1058-66.

19. Agrawal A et al. The genetics of addiction - a translational perspective. Transcult Psychiatry. 2012;2:e140. 
20. Cao J, Hudziak JJ, Li D. Multi-cultural association of the serotonin transporter gene (SLC6A4) with substance use disorder. Neuropsychopharmacology. 2013;38(9):1737-47.

21. Lubke $\mathrm{G}$ et al. The CHRNA5/A3/B4 gene cluster and tobacco, alcohol, cannabis, inhalants and other substance use initiation: replication and new findings using mixture analyses. Behav Genet. 2012;42(4):636-46.

22. Young-Wolff KC, Enoch M-A, Prescott CA. The influence of gene-environment interactions on alcohol consumption and alcohol use disorders: a comprehensive review. Clin Psychol Rev. 2011;31(5):800-16.

23. Dick DM, Kendler KS. The impact of gene-environment interaction on alcohol use disorders. Alcohol Res Curr Rev. 2012;34(3):318-24.

24. Moffitt TE, Caspi A, Rutter M. Measured gene-environment interactions in psychopathology: concepts, research strategies, and implications for research, intervention, and public understanding of genetics. Perspect Psychol Sci. 2006;1(1):5-27.

25. Rutter M, Moffitt TE, Caspi A. Gene-environment interplay and psychopathology: multiple varieties but real effects. J Child Psychol Psychiatry. 2006;47(3-4):226-61.

26. Meyers JL, Dick DM. Genetic and environmental risk factors for adolescent-onset substance use disorders. Child Adolesc Psychiatr Clin N Am. 2010;19(3):465-77.

27. Plomin R, DeFries JC, Knopik VS, Neiderhiser JM. Behavioral genetics. 6th ed. New York: Worth Publishers; 2013.

28. Moffitt TE, Caspi A, Rutter M. Strategy for investigating interactions between measured genes and measured environments. Arch Gen Psychiatry. 2005;62(5):473-81.

29. Tsuang MT et al. Co-occurrence of abuse of different drugs in men: the role of drug-specific and shared vulnerabilities. Arch Gen Psychiatry. 1998;55(11):967-72.

30. Kendler KS et al. Specificity of genetic and environmental risk factors for use and abuse/dependence of cannabis, cocaine, hallucinogens, sedatives, stimulants, and opiates in male twins. Am J Psychiatry. 2003;160(4):687-95.

31. Kendler KS, Myers J, Prescott CA. Specificity of genetic and environmental risk factors for symptoms of cannabis, cocaine, alcohol, caffeine, and nicotine dependence. Arch Gen Psychiatry. 2007;64(11):1313-20.

32. Boardman JD et al. Population composition, public policy, and the genetics of smoking. Demography. 2011;48(4):1517-33.

33. Boardman JD. State-level moderation of genetic tendencies to smoke. Am J Public Health. 2009;99(3):480-6.

34. Boardman JD et al. Do schools moderate the genetic determinants of smoking? Behav Genet. 2008;38(3):234- 46.

35. Dick DM et al. Parental monitoring moderates the importance of genetic and environmental influences on adolescent smoking. $\mathrm{J}$ Abnorm Psychol. 2007;116(1):213-8.

36. Agrawal A et al. Peer substance involvement modifies genetic influences on regular substance involvement in young women. Addiction. 2010;105(10):1844-53.

37. Kendler KS et al. Genetic and family and community environmental effects on drug abuse in adolescence: a Swedish national twin and sibling study. Am J Psychiatry. 2014;171(2):209-17.

$38 . \bullet$ Kendler KS et al. Within-family environmental transmission of drug abuse: a Swedish national study. JAMA Psychiatry. 2013;70(2):235-42. Taking a novel approach to the study of genetic effects, this study provides an unprecedently large sample for analysis. What is striking is that this methodology overcomes a number of the limitations of traditional heritability studies (albeit bringing new study limitations), but findings support those of more traditional designs.

39. Kendler KS et al. Environmental influences on familial resemblance for drug abuse in first-cousin pairs: a Swedish national study. Psychol Med. 2014;44(2):371-9.
40. Giordano GN et al. Unexpected adverse childhood experiences and subsequent drug use disorder: a Swedish population study (19952011). Addiction. 2014;109(7):1119-27.

41. Kendler KS et al. The causal nature of the association between neighborhood deprivation and drug abuse: a prospective national Swedish co-relative control study. Psychol Med. 2014;44(12): 2537-46.

42. Kendler KS et al. Peer deviance, parental divorce, and genetic risk in the prediction of drug abuse in a nationwide Swedish sample: evidence of environment-environment and gene-environment interaction. JAMA Psychiatry. 2014;71(4):439-45.

43. Heath $\mathrm{AC}$ et al. Gene-environment interaction effects on behavioral variation and risk of complex disorders: the example of alcoholism and other psychiatric disorders. Twin Res. 2002;5(1):30-7.

44.• Waldron $\mathrm{M}$ et al. Parental separation and early substance involvement: results from children of alcoholic and cannabis dependent twins. Drug Alcohol Depend. 2014;134:78-84. This study utilizes the Children of Twins design, which avoids some limitations of the classical twin design and allows consideration of potential gene by environment interaction. This paper demonstrates the importance of parental separation as a risk factor over and above the genetic and environmental effects of parental drug dependence.

45. Uher R. Gene-environment interactions in common mental disorders: an update and strategy for a genome-wide search. Soc Psychiatry Psychiatr Epidemiol. 2014;49(1):3-14.

46. Duncan LE, Keller MC. A critical review of the first 10 years of candidate gene-by-environment interaction research in psychiatry. Am J Psychiatry. 2011;168(10):1041-9.

47. Daw $\mathrm{J}$ et al. The interactive effect of neighborhood peer cigarette use and 5HTTLPR genotype on individual cigarette use. Addict Behav. 2014;39(12):1804-10.

48. Laucht $\mathrm{M}$ et al. Catechol-O-methyltransferase Val158 Met genotype, parenting practices and adolescent alcohol use: testing the differential susceptibility hypothesis. J Child Psychol Psychiatry. 2012;53(4):351-9.

49. van der Zwaluw CS et al. Different trajectories of adolescent alcohol use: testing gene-environment interactions. Alcohol Clin Exp Res. 2014;38(3):704-12.

50. Olfson E et al. An ADH1B variant and peer drinking in progression to adolescent drinking milestones: evidence of a gene-byenvironment interaction. Alcohol Clin Exp Res. 2014;38(10): 2541-9.

51. Olsson CA et al. Gene-environment interaction in problematic substance use: interaction between DRD4 and insecure attachments. Addict Biol. 2013;18(4):717-26.

52.• Brody GH, Chen YF, Beach SR. Differential susceptibility to prevention: GABAergic, dopaminergic, and multilocus effects. J Child Psychol Psychiatry. 2013;54(8):863-71. The differential susceptibility hypothesis proposes that, as well as genetic influences priming individuals for greater reactivity to negative environmental exposures, such as exposure to trauma, these genetic influences may also confer greater susceptibility to positive environmental exposures, including treatment and other interventions. This paper is one of the first to study this in the context of interventions for adolescent substance use.

53. Belsky J, Pluess M. Beyond diathesis stress: differential susceptibility to environmental influences. Psychol Bull. 2009;135(6):885908.

54. Sher KJ et al. Consilient research approaches in studying gene $\times$ environment interactions in alcohol research. Addict Biol. 2010;15(2):200-16.

55. Dick DM et al. How phenotype and developmental stage affect the genes we find: GABRA2 and impulsivity. Twin Res Hum Genet. 2013;16(3):661-9. 
56. Kapoor $\mathrm{M}$ et al. Genome-wide survival analysis of age at onset of alcohol dependence in extended high-risk COGA families. Drug Alcohol Depend. 2014;142:56-62.

57. Manolio TA et al. Finding the missing heritability of complex diseases. Nature. 2009;461(7265):747-53.

58. Belsky DW et al. Polygenic risk and the developmental progression to heavy, persistent smoking and nicotine dependence: evidence from a 4-decade longitudinal study. JAMA Psychiatry. 2013;70(5):534-42. This study is one of the few to address the stage-sequential nature of drug use while incorporating a polygenic risk score. Results suggested that genetic risk was unrelated to smoking initiation, but was related to conversion to regular smoking, speed of transition to regular smoking, and cessation failure.

59. McGeary JE et al. Predictors of relapse in a bupropion trial for smoking cessation in recently-abstinent alcoholics: preliminary results using an aggregate genetic risk score. Subst Abus. 2012;6: 107-14.

60. Salvatore JE et al. Polygenic scores predict alcohol problems in an independent sample and show moderation by the environment. Genes (Basel). 2014;5(2):330-46.

61. Wray NR et al. Pitfalls of predicting complex traits from SNPs. Nat Rev Genet. 2013;14(7):507-15.

62. Vrieze SI, McGue M, Iacono WG. The interplay of genes and adolescent development in substance use disorders: leveraging findings from GWAS meta-analyses to test developmental hypotheses about nicotine consumption. Hum Genet. 2012;131(6):791-801. This paper tests whether SNPs are specific to smoking or are relevant for more pervasive behavioural systems. Previous research has found no association between genes associated with smoking initiation and genes associated with cigarettes smoked per day, and this paper finds that nicotine-specific genetic risk becomes more important in adulthood than in adolescence. Effect sizes are small, but the paper highlights important concepts.

63. Hall WD, Mathews R, Morley KI. Being more realistic about the public health impact of genomic medicine. PLoS Med. 2010;7(10): e1000347. doi:10.1371/journal.pmed.1000347

64. Heath AC et al. Estimating two-stage models for genetic influences on alcohol, tobacco or drug use initiation and dependence vulnerability in twin and family data. Twin Res. 2002;5(2):113-24.

65. Nelson EC et al. ANKK1, TTC12, and NCAM1 polymorphisms and heroin dependence: importance of considering drug exposure. JAMA Psychiatry. 2013;70(3):325-33.

66. Kendler KS et al. A population-based twin study in women of smoking initiation and nicotine dependence. Psychol Med. 1999;29(2):299-308.

67. Sartor CE et al. Genetic and environmental influences on the rate of progression to alcohol dependence in young women. Alcohol Clin Exp Res. 2008;32(4):632-8.

68. Sartor CE et al. The role of childhood risk factors in initiation of alcohol use and progression to alcohol dependence. Addiction. 2007;102(2):216-25.
69. Distel MA et al. Age moderates non-genetic influences on the initiation of cannabis use: a twin-sibling study in Dutch adolescents and young adults. Addiction. 2011;106(9):1658-66.

70. Heath AC et al. Genetic and social determinants of initiation and age at onset of smoking in Australian twins. Behav Genet. 1999;29(6):395-407.

71. Kendler KS et al. Recent advances in the genetic epidemiology and molecular genetics of substance use disorders. Nat Neurosci. 2012;15(2):181-9.

72. Kendler KS et al. Genetic and environmental risk factors in the aetiology of illicit drug initiation and subsequent misuse in women. Br J Psychiatry. 1999;175:351-6.

73. Neale MC et al. Extensions to the modeling of initiation and progression: applications to substance use and abuse. Behav Genet. 2006;36(4):507-24.

74. Gillespie NA, Neale MC, Kendler KS. Pathways to cannabis abuse: a multi-stage model from cannabis availability, cannabis initiation and progression to abuse. Addiction. 2009;104(3):430-8.

75. Laucht $\mathrm{M}$ et al. Novelty seeking involved in mediating the association between the dopamine D4 receptor gene exon III polymorphism and heavy drinking in male adolescents: results from a high-risk community sample. Biol Psychiatry. 2007;61(1):87-92.

76. Dupont $\mathrm{C}$, Armant DR, Brenner CA. Epigenetics: definition, mechanisms and clinical perspective. Semin Reprod Med. 2009;27(5): 351-7.

77. Wong CC, Mill J, Fernandes C. Drugs and addiction: an introduction to epigenetics. Addiction. 2011;106(3):480-9.

78. van der Knaap LJ et al. Catechol-O-methyltransferase gene methylation and substance use in adolescents: the TRAILS study. Genes Brain Behav. 2014;13(7):618-25.

79. Nielsen DA et al. Epigenetics of drug abuse: predisposition or response. Pharmacogenomics. 2012;13(10):1149-60.

80. Essex MJ et al. Epigenetic vestiges of early developmental adversity: childhood stress exposure and DNA methylation in adolescence. Child Dev. 2013;84(1):58-75.

81. Dube SR et al. Childhood abuse, neglect, and household dysfunction and the risk of illicit drug use: the adverse childhood experiences study. Pediatrics. 2003;111(3):564-72.

82. Anda RF et al. Adverse childhood experiences and smoking during adolescence and adulthood. JAMA. 1999;282(17):1652-8.

83. Dube SR et al. Adverse childhood experiences and the association with ever using alcohol and initiating alcohol use during adolescence. J Adolesc Health. 2006;38(4):444.e1-e10.

84. Dick DM et al. Genetic influences on alcohol use across stages of development: GABRA2 and longitudinal trajectories of drunkenness from adolescence to young adulthood. Addict Biol. 2014;19(6):1055-64.

85. Sartor CE et al. Common genetic contributions to alcohol and cannabis use and dependence symptomatology. Alcohol Clin Exp Res. 2010;34(3a):545-54. 